\title{
ANÁLISE COMPARATIVA DE ÍNDICES DE QUALIDADE DA ÁGUA SUBTERRÂNEA
}

\author{
Clélia Nobre de Oliveira ${ }^{1}$; Sérgio Augusto de Morais Nascimento ${ }^{2}$; Vânia Palmeiras Campos ${ }^{3}$
}

\begin{abstract}
Resumo - Esse Trabalho analisou alguns índices de qualidade de água (IQAs) e buscou distinguir suas características em função das condições de qualidade do ambiente aquático, interferências naturais e antrópicas, tipos de parâmetros utilizados, normas de referência, usos preponderantes da água e objetivos de aplicação. O IQA da Fundação Nacional de Saneamento, dos Estados Unidos, destaca-se pela sua ampla divulgação e por servir de base para a criação de outros IQAs. O IQA do Conselho Canadense de Ministros do Meio Ambiente, destaca-se pela grande variedade de parâmetros que podem ser inseridos, com os respectivos valores de referência existentes, independente do uso previsto para a água. O Índice de Qualidade Natural de Água SubterrâneaIQNAS, apresenta limitação quanto aos parâmetros usados. O IQA mais utilizado na França se diferencia dos demais ao trabalhar com dezessete grupos de alteração e agregar os parâmetros em função da atividade produtiva ou em função do uso da água. Verificam-se similaridades entre os índices quanto a parâmetros utilizados e que a criação de índices é função das necessidades de cada órgão gestor.
\end{abstract}

Palavras-Chave - IQA; águas subterrâneas; qualidade da água.

\begin{abstract}
This work examined some water quality indices (WQI) and sought to distinguish its characteristics depending on the quality conditions of the aquatic environment, natural and anthropic interference, types of used parameters, reference standards, predominant uses of water and application objectives. The WQI from National Sanitation Foundation of United States stands out for its wide dissemination and serve as a basis for creating other WQI. The WQI from Canadian Council of Ministers of the Environment stands out for the variety of parameters that can be entered, with their existing reference values, regardless of the intended use of the water. The Index of Natural Quality of Groundwater-IQNAS present limitation on the parameters used. The WQI most used in France differs from the others to work with seventeen change groups and add parameters as a function of productive activity or due to the use of water. Occur similarities between the indices as the parameters and the creation of indexes is a function of the needs of each agency manager.
\end{abstract}

Keywords - IQA; groundwater; water quality

\footnotetext{
${ }^{1}$ Núcleo de Estudos Hidrogeológicos e do Meio Ambiente, Instituto de Geociências, Universidade Federal da Bahia, Campus Universitário de Ondina, s/n, 40170290, Salvador-BA, Brasil, (71)3283-8637, clelianobre@ hotmail.com

${ }^{2}$ Departamento de Geofísica, Instituto de Geociências, Universidade Federal da Bahia. Campus Universitário de Ondina, s/n, 40170290, SalvadorBA, Brasil, (71) 3283-8587, sergiomn@ufba.br

${ }^{3}$ Departamento de Química Analítica, Instituto de Química, Universidade Federal da Bahia. Campus Universitário de Ondina, s/n, 40170115, Salvador-BA, Brasil, (71) 3283-6827, vaniaroc@ufba.br
} 


\section{1 - INTRODUÇÃO}

As regiões que utilizam as águas subterrâneas como fonte de água potável possuem grande interesse no desenvolvimento de índices de qualidade da água, visto que o uso desta ferramenta permite reduzir o número de medições necessárias para caracterizar um corpo hídrico, assim como simplificar o processo de informação dos níveis desejados de qualidade da água com o usuário final. Por serem mais protegidas de contaminação, os riscos dos danos à saúde são mais baixos com o uso de águas subterrâneas, o que as tornam mais qualificadas para consumo humano, além de demandarem tecnologias mais simples de tratamento. Cabe ressaltar também os volumes de água potencialmente distribuídos no mundo, que embora de forma desigual, são maiores que os da água superficial, uma vez que "se inter-relacionam com o ciclo hidrológico e dependem das condições climáticas" (SHIKWMANOV, 1998). Segundo a WWAP (2015), cerca de 2,5 bilhões de pessoas no mundo utilizam água subterrânea, com atendimento para consumo humano à pelo menos 50\% da população e é responsável por $43 \%$ de toda a água usada para irrigação.

Há uma projeção que em 2050, 3,9 bilhões de pessoas - de um total de mais de $40 \%$ da população mundial - tendem a viver em bacias hidrográficas sob grande estresse hídrico (OECD, 2012).

Os estudos de monitoramento das águas subterrâneas se tornam emergentes uma vez que o uso dessas águas se encontra nas metas de implementações dos gestores públicos em áreas que a utilizam como fonte de água potável (SAEEDI et al, 2010). Logo, a utilização de índices de avaliação da qualidade da água subterrânea (IQAs) como ferramentas de padronização, agregação e de comunicação do monitoramento da qualidade da água deve ser priorizado nesses casos.

Um IQAs é um uma ferramenta matemática simples que permite integrar dados complexos de qualidade das águas subterrâneas, transformando-os em uma variação de valores numéricos, sintéticos, padronizados e fáceis de interpretação (SUNGSITTHISAWAD \& PITAKSANURAT, 2013), sendo um guia para identificação de alteração na qualidade da água e por conseguinte direcionar seu uso, definindo-se inclusive o melhor processo de tratamento para adequação ao uso pretendido.

A aplicação de índices de qualidade da água subterrânea (IQAs) é evidenciada em pesquisas técnicas e científicas de vários autores que desenvolvem estudos para a melhoria da gestão do monitoramento dos recursos hídricos. Muitos desses autores basearam-se no IQA desenvolvido por Brow et al (1970), o qual foi melhorado por Deininger (1975), conforme descreve Saeedi et al 
(2010). O índice é o Water Quality Index - WQI, criado pela National Sanitation Foundation - NSF (LUMB et al, 2011) e o mais utilizado pela maioria dos países.

Ao longo dos anos, muitos índices foram calculados para fins especiais. Saeedi et al (2010) fez um estudo de aplicação de IQA em águas subterrâneas, no Irã, considerando que são águas com poucos agentes nocivos à saúde. Nesse estudo relaciona autores como: Prati et al (1971), O'Connor (1972), Harkins (1974), Walski e Parker (1974), Inhaber (1975), Shaefer e Janardan (1977), Provencher e Lamontagne (1979), Couillard e Lefebvre (1985) e House e Ellis (1987), os quais calcularam seus índices considerando condições particulares da região de estudo, ou seja, alterando pesos atribuídos a cada situação de risco de contaminação a ser avaliada. Backman et al (1998) apresentou uma evolução no IQAs com um índice para avaliar e mapear o grau de contaminação das águas subterrâneas e testar a sua aplicabilidade no sudoeste da Finlândia e da Eslováquia Central.

Com vistas a satisfazer as várias utilizações da água, o governo francês, através da Agência de Água e do Ministério da Ecologia e Desenvolvimento Sustentável, criou um IQAs (FRANÇA, 2002), hoje considerado ferramenta de referência nacional, pois faz com que seja possível definir a aptidão de uma água para satisfazer as várias utilizações de acordo com os usos prioritários.

No Canadá, um novo índice de qualidade da água, foi desenvolvido, em 2001, o IQA-CCME (Canadian Council of Ministers of the Environment) (CCME, 2001), divergindo do IQA-NSF, modelo dos EUA. O modelo canadense agregou três fatores de análise: alcance, frequência e amplitude, para serem considerados nas comparações com o número de análises realizadas e com os desvios dos padrões de referência.

No Brasil, foram identificados pelo menos 3 IQAs adaptados para avaliação da água subterrânea: Índice de Qualidade Natural de Água Subterrânea - IQNAS (Oliveira et al, 2006, 2007), Índice de Qualidade da Água Subterrânea - IQAS (Oliveira et al, 2004) e Índice de Qualidade de Uso da Água Subterrânea - e-IQUAS (Almeida, 2012). São índices criados com finalidades específicas - análise de água bruta com fins de definição de usos múltiplos, principalmente para atender demandas humanas. A fundamentação desses modelos é o IQA-NSF, mas com as devidas adequações para as características dos domínios hidrogeológicos brasileiros, o que determina a relevância dos mesmos para melhorar o processo de monitoramento das águas subterrâneas.

Assim observa-se com este levantamento bibliográfico que existe uma variedade de IQAs criados para atender situações específicas de cada região em algumas partes do mundo. Desta forma este trabalho se propôs a realizar uma análise comparativa de alguns destes índices de qualidade da água utilizados para avaliação de águas subterrâneas, procurando distinguir as peculiaridades de cada um em função de sua aplicação, condições da qualidade do recurso hídrico, interferências 
naturais e antrópicas, número e tipos de parâmetros utilizados, normas de referência (padrões nacionais e internacionais), usos preponderantes da água e objetivos de aplicação.

\section{2 - RESULTADOS E DISCUSSÃO}

\section{1 - Índice WQI-NSF / USA}

O índice WQI-NSF (Water Quality Index-National Sanitation Foundation) ou IQA-NSF (Índice de Qualidade da Água - Fundação Nacional de Saneamento), é considerado como o de maior aplicação, incialmente nos Estados Unidos, desde 1976, após adaptações, sendo o país de criação, como por outros países que perceberam a importância dessa ferramenta para monitoramento das águas subterrâneas, já em processo intenso de utilização para atender demandas de consumo humano (YISA et al, 2012).

Após adaptações, gerou-se o IQA multiplicativo (Equação 1), com atribuição de pesos específicos (Quadro 1) para cada um dos nove parâmetros que o compõe: oxigênio dissolvido, coliformes termotolerantes, potencial hidrogeniônico, demanda bioquímica de oxigênio, nitratos, fosfatos, temperatura, turbidez e sólidos totais dissolvidos. O peso de cada parâmetro resultou em curvas médias representativas da variação da qualidade da água em função das suas respectivas concentrações (YISA et al, 2012).

Quadro 1. Parâmetros utilizados no IQA-NSF, com respectivos pesos

\begin{tabular}{|c|c|}
\hline Parâmetro & Peso (wi) \\
\hline Oxigênio Dissolvido (OD) & 0,17 \\
\hline Coliformes Termotolerantes (CTe) & 0,16 \\
\hline Potencial Hidrogeniônico $(\mathrm{pH})$ & 0,11 \\
\hline Demanda Bioquímica de Oxigênio (DBO) & 0,11 \\
\hline Nitratos $\left(\mathrm{NO}_{3}\right)$ & 0,10 \\
\hline Fosfatos $\left(\mathrm{PO}_{4}\right)$ & 0,10 \\
\hline Temperatura $(\mathrm{T})$ & 0,10 \\
\hline Turbidez (Turb) & 0,08 \\
\hline Sólidos Totais Dissolvidos (STD) & 0,07 \\
\hline$\Sigma$ & 1,0 \\
\hline
\end{tabular}

Fonte: YISA et al, 2012.

Os noves parâmetros foram selecionados a partir de pesquisas realizadas com vários especialistas da área ambiental.

A formulação final proposta por Deininger \& Landwehr (1976) apud Almeida (2012) resultou na forma multiplicativa com os pesos tornando-se potências dos subíndices, conforme Equação 1.

$$
\mathrm{IQA}_{-\mathrm{NSF}} 1976=\pi^{\mathrm{n}} \mathrm{qi}^{\mathrm{wi}}
$$

Onde: 
IQA-NSF 1976: representa um número de 0 a 100;

qi: subíndice ou qualidade do i-ésimo parâmetro (entre 0 e 100), obtido através da curva média de variação de qualidade em função da concentração;

$\mathrm{W}_{\mathrm{i}}$ : peso atribuído ao parâmetro, entre 0 e 1, em função de sua importância na qualidade.

A partir desses sub-índices se definiu faixas de qualidade da água (Quadro 2) que são utilizadas para definir o resultado do IQA, a partir de 5 tipos de classificação:

Quadro 2. Nível de qualidade ou classificação da água em função do resultado do IQA-NSF:

\begin{tabular}{|c|c|}
\hline Nível de Qualidade & Faixa \\
\hline Excelente & $90<\mathrm{IQA} \leq 100$ \\
\hline Bom & $70<\mathrm{IQA} \leq 90$ \\
\hline Médio & $50<\mathrm{IQA} \leq 70$ \\
\hline Ruim & $25<\mathrm{IQA} \leq 50$ \\
\hline Muito ruim & $00<\mathrm{IQA} \leq 25$ \\
\hline
\end{tabular}

Fonte: YISA, 2012

Os sub-índices foram agrupados, formando faixas de 0 a 100, evidenciando-se que as faixas de qualidade acima de 50, determinam melhores condições para a água ser consumida, levando-se em conta que ainda precise ter seus padrões adequados às normas de referência, por exemplo à norma de potabilidade da Organização Mundial de Saúde-OMS (WHO, 2004).

Cabe ressaltar a aplicabilidade desse índice no Brasil a partir de 1975, mas que sofreu adequações para atendimentos das condições de contaminação mais frequentes na maioria dos estados brasileiros, nesse caso por efluentes domésticos, conforme Quadro 3.

Quadro 3. Faixas de classificação da água em função do resultado do IQA da CETESB

\begin{tabular}{|c|c|}
\hline Avaliação da Qualidade & $\begin{array}{c}\text { Faixas de IQA utilizadas nos estados de } \\
\text { BA, CE, ES, GO, MS, PB, PE, SP }\end{array}$ \\
\hline Ótima & $80<\mathrm{IQA} \leq 100$ \\
\hline Boa & $52<\mathrm{IQA} \leq 79$ \\
\hline Razoável & $37<\mathrm{IQA} \leq 51$ \\
\hline Ruim & $20<\mathrm{IQA} \leq 36$ \\
\hline Péssima & $00<\mathrm{IQA} \leq 19$ \\
\hline
\end{tabular}

Fonte: Programa Nacional de Avaliação da Qualidade das Aguas (PNQA), 2016

Atualmente o IQA, adequado pela CETESB é o principal índice de qualidade da água utilizado no país, abrangendo pelo menos 15 estados brasileiros, os quais realizam o monitoramento das águas superficiais para fins de abastecimento público. A partir de 2002, a empresa desenvolveu índices mais específicos, dentre eles o IAP (Índice de Qualidade de Águas Brutas para Fins de Abastecimento Público), o qual atende à tomada de decisão do uso da água de rios e reservatórios antes de passar pelo processo de tratamento (PNQA, 2016). 


\section{2 - Índice SEQ-EAUX / FRANÇA}

A análise do índice francês, SEQ-EUAX (systeme d'evaluation de la qualite des eaux souterraines), ou IQA-FRANÇA foi baseada nos estudos da própria Agencia Francesa de Água (FRANÇA, 2002), e nos estudos de CADILHAC \& ALBINET (2003). A elaboração desse índice fez parte do programa de inter-agências criado em 1994, cujo objetivo foi desenvolver um SEQ Eaux souterraines (Sistema de Avaliação da Qualidade de Água Subterrânea) com vistas de harmonizar, modernizar e enriquecer os sistemas de avaliação da qualidade dos ambientes aquáticos. Este grupo, liderado pela Agência de Água do pais, foi composto por representantes de outros órgãos, também ligados à gestão da água e meio ambiente.

A ideia era definir o atendimento aos vários usos da água, em função da sua importância, criando um índice que deveria expressar a magnitude das alterações físico-químicas da água em uma determinada região que sofre alterações devido à presença de atividades humanas. Para a classificação da qualidade água (Quadro 4), foram definidas de 3 a 5 classes, com adoção de cores (azul, verde, amarelo, laranja e vermelho).

Quadro 4. Cores e classes de qualidade da água - modelo do IQA francês

\begin{tabular}{|c|c|c|}
\hline Classe/cores & Índice & Classe de qualidade \\
\hline Azul & 80 a 100 & muito boa qualidade \\
\hline Verde & 60 à 79 & boa qualidade \\
\hline Amarelo & 40 à 59 & qualidade regular \\
\hline Laranja & 20 a 39 & qualidade ruim \\
\hline Vermelho & 0 à 19 & qualidade muito ruim \\
\hline
\end{tabular}

Fonte: Adaptado de CADILHAC \& ALBINET (2003)

O IQA-FRANÇA avalia os grupos de alteração de acordo com o uso e finalidade da água, variando de 0 a 100.

Os dezessete grupos foram definidos por tipo de poluição e em função disso se definem os parâmetros que indicam a presença dos níveis de ocorrência na água (Quadro 5):

Quadro 5. Definição dos grupos de alterações na água - IQA-FRANÇA

\begin{tabular}{|c|c|c|}
\hline \multicolumn{3}{|c|}{ Grupos de Alterações } \\
\hline 1- Sabor e Odor & 7 - Micropoluentes Minerais & 13 - Mineralização e Salinidade \\
\hline 2 - Pesticidas & 8 - Material Orgânico e Oxidável & 14 - Nitrato \\
\hline $\begin{array}{l}3 \text { - Partículas em } \\
\text { Suspensão }\end{array}$ & $\begin{array}{l}9 \text { - Hidrocarbonetos Aromáticos } \\
\text { Policíclicos (HAP) }\end{array}$ & 15 - Formação de Depósitos \\
\hline 4 - Ferro e Manganês & 10 - Policlorobifenil (PCB) & 16 - Temperatura \\
\hline 5 - Coloração & 11 - Micropoluentes Orgânicos (outros) & $\begin{array}{l}17 \text { - Nitrogenados (exceto } \\
\text { Nitrato) }\end{array}$ \\
\hline 6 - Microorganismos & 12 - Corrosão & \\
\hline
\end{tabular}

Fonte: CADILHAC \& ALBINET (2003) apud MENEZES (2009) 
Em função da definição do parâmetro pertencentes a cada grupo, são atribuídos valores para esses grupos e definidas as classes de adequação.

A formulação matemática do SEQ - Eaux Souterraines, também adota o método do IQANSF. Cadilhac \& Albinet (2003), ressaltam que o modelo foi construído para atender as normas europeias e francesas e que a estrutura do IQA-FRANÇA é flexível e permite a inserção de novos parâmetros a qualquer momento.

\section{3 - Índice WQI-CCME / CANADA}

O índice WQI-CCME (Water Quality Index-Canadian Council of Ministers of the Environment) ou IQA-CCME (Índice de Qualidade da Água-Conselho Canadense de Ministros do Meio Ambiente), fornece resultados expressos em faixas de valores, que determinam o nível de qualidade da água. De acordo com Hurley et al (2012), sua criação baseou-se no IQA-NSF, e após adequações, em 2001, tornou-se um índice flexível, adaptável às condições locais, devido às suas especificidades, podendo ser utilizado para determinar o nível de tratamento de uma água de fonte potável.

O IQA-CCME é um índice que compara os resultados das análises da água com níveis de qualidade, cuja pontuação varia de 0 , representando a pior qualidade, até 100 , representando a melhor qualidade.

O IQA-CCME é calculado da seguinte forma:

$$
\mathrm{IQA}-\mathrm{CCME}=100-\left[\frac{\sqrt{\mathrm{F}^{2}+\mathrm{F} 2^{2}+\mathrm{F} 3^{2}}}{1,732}\right]
$$

onde:

F1 (\%) (alcance): percentual que representa quantas vezes a variável (parâmetro) analisada se apresenta fora do limite da norma em referência, pelo menos uma vez durante o período de tempo considerado;

F2 (\%) (frequência): percentual que representa o número de vezes que cada amostra analisada apresenta o parâmetro fora do limite, no período de tempo considerado;

F3 (\%) (amplitude): percentual que representa o desvio de cada parâmetro em relação ao seu respectivo limite de referência.

O divisor 1,732 normaliza os valores resultantes para um intervalo entre 0 e 100 , onde 0 representa a "pior" qualidade da água e 100 representa a "melhor" qualidade da água (CCME, 2001). 
A classificação do IQA-CCME (Quadro 6) obedece às seguintes categorias:

Quadro 6. Categorias de classificação da qualidade da água - IQA-CCME

\begin{tabular}{|c|c|c|}
\hline $\begin{array}{l}\text { Nível de Qualidade } \\
\text { / Categoria }\end{array}$ & Faixa & Interpretação \\
\hline Excelente & $95-100$ & $\begin{array}{l}\text { água protegida, com uma virtual ausência de ameaça ou } \\
\text { prejuízo; condições muito próximas dos níveis naturais ou } \\
\text { intocada. }\end{array}$ \\
\hline Bom & $80-94$ & $\begin{array}{c}\text { água protegida, com baixa possibilidade de ameaça ou } \\
\text { prejuízo; condições próximas dos níveis naturais ou } \\
\text { desejáveis. }\end{array}$ \\
\hline Regular & $65-79$ & $\begin{array}{c}\text { água geralmente protegida, mas, ocasionalmente, ameaçada ou } \\
\text { prejudicada; condições, por vezes, afasta-se dos níveis } \\
\text { naturais ou desejáveis. }\end{array}$ \\
\hline Ruim & $45-64$ & $\begin{array}{l}\text { água frequentemente ameaçada ou prejudicada; condições } \\
\text { muitas vezes fora dos níveis naturais ou desejáveis. }\end{array}$ \\
\hline Muito ruim & $0-44$ & $\begin{array}{l}\text { água quase sempre ameaçada ou prejudicada; condições } \\
\text { geralmente fora de níveis naturais ou desejáveis }\end{array}$ \\
\hline
\end{tabular}

Tyagi et al (2013), fazem críticas favoráveis que subsidiam a utilização do modelo pelos órgãos gestores, pois apresenta como resultado a indicação dos usos permissíveis para a água, o que já facilita na tomada de decisão. Já as análises desfavoráveis indicam oportunidades de melhorias, para a adequação do modelo, principalmente no que se refere ao tratamento das variáveis, que apresentam resultados às vezes relevantes para identificar um risco de contaminação específico, mas devido à padronização exigida no modelo, alguma determinada variável que apresente um desvio significativo não é evidenciada nos resultados da simulação.

\section{4 - Índice IQNAS / BRASIL}

O índice IQNAS (Índice de Qualidade Natural de Água Subterrânea), desenvolvido por pesquisadores da Universidade Federal da Bahia (UFBA), em 2004, foi criado principalmente para transmitir as peculiaridades locais da água subterrânea do estado brasileiro. Por conta disso, Oliveira et al (2006), reforça que o IQNAS é uma ferramenta de grande utilidade para o público em geral, contribuindo para a realização da avaliação sazonal da qualidade da água em diversos pontos de um mesmo corpo d'água, como também em corpos d'água diferentes, permitindo uma análise comparativa e com isso obtenção da classificação do nível de qualidade do manancial, acompanhado dos possíveis usos.

Assim como o SEQ, a metodologia de criação do IQNAS, se assemelha ao método do IQA proposto pela NSF (National Sanitation Foundation), já descrito anteriormente. 
O IQNAS foi desenvolvido conforme três estratégias básicas: i) levantamento da opinião de especialistas, ii) utilização de dados hidroquímicos, e, iii) aplicação de métodos estatísticos (OLIVEIRA et al, 2006).

O IQNAS utiliza parâmetros químicos significativos para se avaliar a qualidade da água de domínios hidrogeológicos do Estado da Bahia (sedimentar, metassedimentar, cárstico e cristalino): cloreto, $\mathrm{pH}$, resíduos totais, dureza, flúor e nitrato. Almeida (2012) ressalta que "por abranger apenas cátions e ânions mais importantes, o IQNAS é voltado para a qualidade natural das águas”.

A formulação matemática (Eq. 3) escolhida para o IQNAS foi a mesma utilizada para o IQA da CETESB:

$$
\begin{aligned}
\text { IQNAS } & =\mathrm{Q}_{1}{ }^{\mathrm{w} 1} \times \mathrm{Q}_{2}{ }^{\mathrm{w} 2} \times \mathrm{Q}_{3}{ }^{\mathrm{w} 3} \times \ldots . . \mathrm{Q}_{\mathrm{n}}{ }^{\mathrm{wn}} ; \\
\text { IQNAS } & =\pi^{\mathrm{n}} \mathrm{Qi}^{\mathrm{wi}}
\end{aligned}
$$

Ou seja, um produto dos valores de qualidade da água subterrânea para cada parâmetro químico escolhido $\left(\mathrm{Q}_{\mathrm{i}}\right)$, elevado ao peso atribuído a cada variável ( $\left.\mathrm{w}_{\mathrm{i}}\right)$.

Os pesos $\left(\mathrm{w}_{\mathrm{i}}\right)$ propostos por especialistas em hidrogeologia do Estado da Bahia foram testados utilizando-se funções matemáticas estabelecidas para cada parâmetro (Quadro 7); as notas finais $\left(\mathrm{Q}_{\mathrm{i}}\right)$ foram definidas realizando-se a relação dos resultados das concentrações de cada parâmetro nas respectivas curvas de qualidade, tomando como base a legislação de potabilidade vigente. A construção das curvas características da qualidade relativa de cada parâmetro pode ser conferida em Oliveira et al. (2007).

Quadro 7. Parâmetros utilizados no IQNAS, com respectivos pesos

\begin{tabular}{|l|c|}
\hline Parâmetro & Peso (wi) \\
\hline Cloreto & 0,26 \\
\hline $\mathrm{pH}$ & 0,05 \\
\hline Resíduos totais & 0,22 \\
\hline Dureza & 0,16 \\
\hline Flúor & 0,16 \\
\hline Nitrato & 0,15 \\
\hline & $\Sigma$ \\
\hline
\end{tabular}

Fonte: OLIVEIRA et al. (2007)

As funções matemáticas estabelecidas com as notas de qualidade adotadas ( 0 a 100), foram testadas no IQA-NSF 1976, adequando-se as seguintes faixas de notas para o IQNAS: notas de 0 a 36; de 37 a 51; de 52 a 79 e 80 a 100, variando de imprópria a ótima, respectivamente.

Na análise da aplicação do IQNAS, Menezes (2009), destaca que a inserção dos parâmetros Cloreto e Resíduos totais induzem à resultados redundantes ou multicolineares. Como a principal função dos indicadores de qualidade da água é evidenciar a presença de determinada substância na água, essa redundância não afeta o objetivo fim, que é classificar o nível da qualidade, principalmente porque o IQNAS adota faixas de qualidade abrangentes. 


\section{3 - CONCLUSÃO}

Reunindo-se alguns dos pareceres dos autores aqui referenciados, a aplicação de IQAs como ferramenta de avaliação dessas águas é primordial para dar subsídio à gestão dos recursos hídricos quanto se trata de tomar decisão quanto aos seus usos, principalmente se for para atender consumo humano.

França, Canadá e Brasil, criaram IQAs que podem permitir uma análise em função do tipo de uso pretendido ou em função da interferencia previamente identificada.

Independente das peculiaridades a serem consideradas no índice, eles são úteis para fins de comparação entre áreas com determinado nível de impacto. Uma área com atividades industriais pode apresentar maior inconformidade para usos de consumo humano, mas pode ser utilizada para outros usos, que não exigem nível de tratamento elevado.

Os IQAs: IQNAS-BRASIL e NSF apresentam limitação quanto ao número de parâmetros usados, de 6 a 9 , respectivamente.

O IQA-CCME destaca-se pela grande variedade de parâmetros que podem ser inseridos, com os respectivos valores de referência existentes, independente do uso da água previsto.

O IQA-FRANÇA, também não tem limite de parâmetros, mas possuí uma padronização diferente ao trabalhar por grupos (17) e agregar os parâmetros em função da atividade produtiva ou em função do uso da água pretendido.

O IQA-NSF (USA), um dos primeiros IQAs a serem aplicados pelos governos, destaca-se pela sua ampla divulgação, e por servir de base para a criação de outros IQAs.

É notório que todos os IQAs são ferramentas de comunicação entre o gestor e o usuário da água e permitem sua análise, principalmente para garantir o uso para consumo humano.

Cabe ressaltar, que o índice a ser utilizado, deve ser cuidadosamente selecionado, pois alguns dos índices existentes, foram criados para atender determinada especificidade de uma região, a exemplo do IQA francês e do brasileiro.

Verifica-se portanto, que atualmente existem esforços no sentido de se criar índices em diversas regiões do planeta, a maioria com fins mais regionais, mas devido à diversidade dos que já foram aplicados, os estudos sinalizam a necessidade de se buscar um IQAs que seja universalmente aceito, uma vez que, devido às suas características hidrogeoquímicas e hidrogeológicas, as águas subterrâneas podem demandar índices específicos, mesmo considerando seu local de ocorrência, comportamento no meio, interferências naturais e antrópicas e disponibilidade. 


\section{4 - REFERÊNCIAS}

ALMEIDA, Rosa A. S. de. (2012). Índice de Qualidade de Uso da Água Subterrânea (e-IQUAS): uma Metodologia de Modelagem Numérica Flexível. Tese de Doutorado (Doutorado em Energia e Ambiente), Escola Politécnica, Universidade Federal da Bahia, UFBA, Salvador, 344 p.

BACKMAN, B., BODIS, D., LAHERMO, P., RAPANT, S., \& TARVAINEN, T. (1998). Application of a groundwater contamination index in Finland and Slovakia. Environmental Geology, 55-64.

CADILHAC, L.; ALBINET, M. (Coord.). (2003). SEQ - Système d'évaluation de la qualité des eaux souterraines: Rapport de présentation. Version 0.1. Agences de 1'Eau. 2003. 75p.

CCME. Canadian Council of Ministers of the Environment. (2001). Canadian water quality guidelines for the protection of Water Quality Index, User's Manual. In: Canadian environmental quality guidelines. Canadian Council of aquatic life: CCME, Winnipeg. Disponível em http://www.ccme.ca/en/resources/canadian_environmental_quality_guidelines/index.html. Acesso em set/2014.

FRANÇA. Agencia de Água. (2002). SYSTEME D’EVALUATION DE LA QUALITE DES EAUX SOUTERRAINE. SEQ - Eaux souterraines - Rapport de présentation, Version 0. Agencia de Água, 1-66.

HURLEY Tim, SADIQ, Rehan, MAZUMDER, Asit. (2012). Adaptation and evaluation of the Canadian Council of Ministers of the Environment Water Quality Index (CCME WQI) for use as an effective tool to characterize drinking source water quality. Journal of the American Water Resources Association 46, 3544 e 3552.

LUMB, A., SHARMA, T.C., BIBEAULT, J. F., KLAWUNN, P. (2011). A Comparative Study of USA and Canadian Water Quality Index Models. Water Qual Expo Health, 203-216.

MENEZES, Juliana Magalhães. (2009). Índice de Qualidade de Água Subterrânea Aplicado em Área de Aquíferos Cristalinos com Uso Agrícola: Bacia do Rio São Domingos-RJ. Tese (doutorado), Universidade Federal do Rio de Janeiro, Programa de Pós Graduação em Geologia, UFRJ/PPG, Rio de Janeiro, 189 p.

OECD. Organização para a Cooperação e Desenvolvimento Econômico. Environmental (2012). Outlook to 2050: The Consequences of Inaction, 2012. Disponível em http://www.oecd.org/env. Acesso em dez/2014.

OLIVEIRA I.B., NEGRÃO, F.I., SILVA, A.G.L.S. (2006). Aplicação do Índice de Qualidade Natural das Águas Subterrâneas - IQNAS, para os Domínios Hidrogeológicos do Estado da Bahia. Anais do XIV Congresso Brasileiro de Águas Subterrâneas. Curitiba, 7-10 Novembro de 2006. 
OLIVEIRA I.B., NEGRÃO, F.I., SILVA, A.G.L.S.(2007). Mapeamento dos Aquíferos do Estado da Bahia Utilizando o Índice de Qualidade Natural das Águas Subterrâneas - IQNAS. Águas Subterrâneas, v.21, n.1, p.123-137.

OLIVEIRA, I. B.; NEGRÃO, F. I.; ROCHA, T. S. (2004). Determinação do Índice de Qualidade da Água Subterrânea - IQAS, com base nos dados de poços tubulares do estado da Bahia: Áreas Piloto: Recôncavo e Platô de Irecê - Cuiabá. Anais do XIII Congresso Brasileiro de Águas Subterrâneas, 19-22 Outubro de 2004.

PNQA. Programa Nacional de Avaliação da Qualidade das Águas. Indicadores de qualidade - índice de qualidade das águas (IQA). (2016). Disponível em: http://portalpnqa.ana.gov.br/ indicadoresindice-aguas.aspx. Acesso em: jan/2016.

SAEEDI, Mohesn; ABESSI, Ozeair; SHARIFI, Farid; MERAJI, Hamed. (2010). Development of groundwater quality index. Environ Monit Assess, 327-335.

SHIKWMANOV, Igor A. (1998). World Water Resources a new appraisal and assessment for the 21st century. State Hydrological Institute, St Petersburg, Russia.

SUNGSITTHISAWAD, Warangkana \& PITAKSANURAT, Somsak. (2013). Groundwater Quality Index for Water Supply Production. Thailandia Society of Higher Education Institutes on Environment. EnvironmentAsia, 18-23.

TYAGI, Shweta, SHARMA, Bhavtosh, SINGH, Prashant, DOBHA, Rajendra. (2013). Water Quality Assessment in Terms of Water Quality Index. American Journal of Water Resources, Vol. 1, No. 3, 34-38.

WHO. World Health Organization. (2004). Guidelines for drinking-Water Quality. Vol. 1, $3^{\mathrm{a}}$ edição. WHO Library Cataloguing-in-Publication Data, Geneva, WHO.

WWAP (United Nations World Water Assessment Programme). (2015). The United Nations World Water Development Report 2015: Water for a Sustainable World. Paris, UNESCO.

YISA, Jonathan; JIMOH, Tijani Oladejo; OYIBO, Ohiemi Michael. (2012). Underground Water Assessment using Water Quality Index. Leonardo Journal of Sciences, July-December, 2012, 33-42. 